\title{
Holographic entanglement entropy for hollow cones and banana shaped regions
}

\author{
Harald Dorn \\ Institut für Physik und IRIS Adlershof, Humboldt-Universität zu Berlin, \\ Zum Großen Windkanal 6, D-12489 Berlin, Germany \\ E-mail: dorn@physik.hu-berlin.de
}

ABSTRACT: We consider banana shaped regions as examples of compact regions, whose boundary has two conical singularities. Their regularised holographic entropy is calculated with all divergent as well as finite terms. The coefficient of the squared logarithmic divergence, also in such a case with internally curved boundary, agrees with that calculated in the literature for infinite circular cones with their internally flat boundary. For the otherwise conformally invariant coefficient of the ordinary logarithmic divergence an anomaly under exceptional conformal transformations is observed.

The construction of minimal submanifolds, needed for the entanglement entropy of cones, requires fine-tuning of Cauchy data. Perturbations of such fine-tuning leads to solutions relevant for hollow cones. The divergent parts for the entanglement entropy of hollow cones are calculated. Increasing the difference between the opening angles of their outer and inner boundary, one finds a transition between connected solutions for small differences to disconnected solutions for larger ones.

Keywords: AdS-CFT Correspondence, Differential and Algebraic Geometry, Gaugegravity correspondence

ARXIV EPRINT: 1602.06756 


\section{Contents}

1 Introduction 1

2 Entanglement entropy for a cone $\quad 3$

3 Stability analysis for the cone solutions and study of hollow cone solutions

4 Elementary geometry of banana shaped regions $\quad 11$

$5 \quad$ Entanglement entropy for banana shaped regions 13

$\begin{array}{lll}6 & \text { Conclusions } & 17\end{array}$

$\begin{array}{ll}\text { A Evaluation of the integrals } J_{1} \text { and } J_{2} & 19\end{array}$

B Anomaly under exceptional conformal transformations for smooth boundaries

\section{Introduction}

For a quantum field theory in $(d+1)$ dimensions the entanglement entropy of a $d$-dimensional spatial region $\mathcal{A}$ is defined by ${ }^{1}$

$$
S(\mathcal{A})=-\operatorname{tr}\left(\rho_{\mathcal{A}} \log \rho_{\mathcal{A}}\right),
$$

where $\rho_{\mathcal{A}}$ is the density operator obtained by integrating out the degrees of freedom outside $\mathcal{A}$. In conformal field theories with a holographic dual, defined in $A d S_{d+2}$ or some of its modifications, $S(\mathcal{A})$ at strong coupling can be related to the volume of the minimal spatial $d$-dimensional submanifold $\gamma_{\mathcal{A}} \subset A d S_{d+2}$, which approaches the boundary $\partial \mathcal{A}$ of $\mathcal{A}$ on the boundary of AdS, by $[1,3]$

$$
S(\mathcal{A})=\frac{V\left(\gamma_{\mathcal{A}}\right)}{4 G_{N}^{(d+2)}}
$$

In this formula $V\left(\gamma_{A}\right)$ is the volume of $\gamma_{\mathcal{A}}$ and $G_{N}^{(d+2)}$ denotes the $(d+2)$-dimensional Newton constant.

Due to the near boundary behaviour of the AdS metric, these volumes are divergent. The standard procedure for a regularisation refers to the use of Poincaré coordinates

$$
d s^{2}=\frac{1}{r^{2}}\left(d r^{2}+d x^{\mu} d x_{\mu}\right)
$$

\footnotetext{
${ }^{1}$ For reviews see e.g. $[1,2]$ and refs. therein.
} 
and cutting off that part of the submanifold whose $r$-coordinate is smaller than $\epsilon$. For smooth $\partial \mathcal{A}$, which by construction is the boundary of $\gamma_{\mathcal{A}}$, the small $\epsilon$-expansion of $V_{\epsilon}\left(\gamma_{\mathcal{A}}\right)$ has the following structure [4]

$$
\begin{array}{ll}
V_{\epsilon}=\frac{c_{1}}{\epsilon^{d-1}}+\frac{c_{3}}{\epsilon^{d-3}}+\ldots+\frac{c_{d-2}}{\epsilon^{2}}+a \log \epsilon+c_{d}+o(1), & \text { for odd } d \\
V_{\epsilon}=\frac{c_{1}}{\epsilon^{d-1}}+\frac{c_{3}}{\epsilon^{d-3}}+\ldots+\frac{c_{d-1}}{\epsilon}+c_{d}+o(1), & \text { for even } d .
\end{array}
$$

Although conformal transformations on the boundary of AdS act as isometries in the bulk, conformal invariance is broken by the use of the cut-off. Nevertheless for odd $d$ the special coefficient $a$ and for even $d$ the special coefficient $c_{d}$ are invariant with respect to conformal transformations of the boundary of $\gamma_{\mathcal{A}}$ [4].

The coefficient of the leading divergence is proportional to the volume of $\partial \mathcal{A}[1]$ and the coefficient of the $\log \epsilon$ term can be expressed as an integral over a conformally invariant quantity constructed out of the second fundamental form of $\partial \mathcal{A}[5]$.

For singular boundaries additional divergences show up. In particular, for isolated conical singularities of $\partial \mathcal{A}$ (cusps in the case $d=2$ ) these new contributions are logarithmic for even $d$ and double logarithmic for odd $d[6]$. Their behaviour in the smooth limit has been related to a certain central charge of the CFT [7]. The minimal surfaces needed for the entanglement entropy in $d=2$ are also relevant for the holographic treatment of the strong coupling behaviour of Wilson loops [8,9]. There the coefficient of the logarithmic divergence, called cusp anomalous dimension, has been calculated in [10]. The corresponding discussion applied to the entanglement entropy can be found in [11, 12].

As in the two-dimensional case, also in higher dimensions the extraction of the coefficient of the additional divergence, generated by a conical singularity, has been performed by choosing $\partial \mathcal{A}$ as the boundary of an infinite circular cone $[6,13]$. It is then expected, that the coefficient remains unchanged if one allows arbitrary curvature in the neighbourhood of the singular point, while keeping the opening angle fixed. We have checked this expectation for the two-dimensional case [15], both by the calculation of the area of the minimal surface related to a curved compact $\partial \mathcal{A}$ obtained by two intersecting circles, as well as by a general proof.

The present paper is devoted to the analogous problem for $d=3$. We will calculate the regularised volume up to terms vanishing for $\epsilon \rightarrow 0$ for the three-dimensional minimal submanifold $\gamma_{\mathcal{A}}$, which reaches the boundary of Euclidean $A d S_{4}{ }^{2}$ and meets there the boundary of a banana shaped region $\mathcal{A} .^{3}$ The construction is technically a bit more involved as in $d=2$, since a helpful conservation law is no longer available, and one has to handle a second order nonlinear differential equation instead of an integrable first order one. As a by-product of our analysis we will find the coefficients of divergences for a new type of singularity of $\partial \mathcal{A}$, the hollow cone.

The paper is organised as follows. To set up some notation we review and comment in section 2 the calculation for the infinite circular cone as performed in [6, 13]. In section 3 we

\footnotetext{
${ }^{2}$ The time coordinate in $A d S_{5}$ is fixed.

${ }^{3}$ To our knowledge it will be the first explicit calculation for a compact region $\mathcal{A}$ with singularities on its boundary $\partial \mathcal{A}$.
} 
study the issue of stability under perturbations of the Cauchy initial data for the differential equation under study. This will bring us solutions for hollow cones, whose inner and outer conical surfaces have a common tip.

Section 4 collects some elementary geometrical properties of certain banana shaped regions, and in section 5 we apply a suitable conformal transformation of cones to get the regularised volume $V_{\epsilon}\left(\gamma_{\mathcal{A}}\right)$ for banana shaped regions $\mathcal{A}$. We conclude in section 6 and have put some technical details related to section 5 into appendix A. The second appendix $\mathrm{B}$ presents facts on an anomaly under exceptional conformal transformations needed in the conclusion section.

\section{Entanglement entropy for a cone}

For the holographic entanglement entropy of a cone in $\mathbb{R}^{3}$ the divergent parts of the relevant volume have been calculated in $[6,13]$. Here we repeat some steps of the calculation, both to set up some notation and to pick up also the finite part.

One has to find the 3-dimensional minimal submanifold in the bulk with Euclidean $A d S_{4}$-metric in Poincaré coordinates, see (1.3), which approaches the boundary of the cone for $r \rightarrow 0$. Using the symmetry of the problem we can make the ansatz

$$
\begin{aligned}
x_{1} & =\rho \sin \vartheta \cos \varphi, \quad x_{2}=\rho \sin \vartheta \sin \varphi, \quad x_{3}=\rho \cos \vartheta \\
r & =\rho h(\vartheta),
\end{aligned}
$$

with $0 \leq \rho<\infty, \quad 0 \leq \varphi<2 \pi, \quad 0 \leq \vartheta \leq \Omega$ and $2 \Omega$ denoting the opening angle of the cone $(0<\Omega<\pi / 2)$. The boundary condition for $h(\vartheta)$ is

$$
h(\Omega)=0 .
$$

The volume of the manifold $(2.1)$ is $(\dot{h}=d h(\vartheta) / d \vartheta)$

$$
V=2 \pi \int_{0}^{\infty} \frac{d \rho}{\rho} \int_{0}^{\Omega} d \vartheta \frac{\sin \vartheta}{h^{3}(\vartheta)} \sqrt{1+h^{2}+\dot{h}^{2}}
$$

It needs both an IR regularisation at large $\rho$ as well as an UV regularisation near the boundary of AdS, i.e $\vartheta=\Omega$ or $\rho=0$.

In the analogous case in $A d S_{3}$ there is no explicit $\vartheta$-dependence in the integrand, and the related conservation law yields a first order differential equation which can be solved by integration [10].

However, here $\vartheta$ appears explicitly, and we are forced to handle the second order differential equation which enforces the stationarity condition for (2.3)

$$
\begin{aligned}
& \left(\ddot{h}\left(h+h^{3}\right)+\dot{h}^{2}\left(3+h^{2}\right)+3+5 h^{2}+2 h^{4}\right) \sin \vartheta \\
& +h \dot{h}\left(1+h^{2}+\dot{h}^{2}\right) \cos \vartheta=0 .
\end{aligned}
$$

This equation is singular at $\vartheta=0$. If one looks for regular solutions at this point, they have to obey either $\dot{h}=0$ or $h=0$, implying that there the initial value problem cannot be 


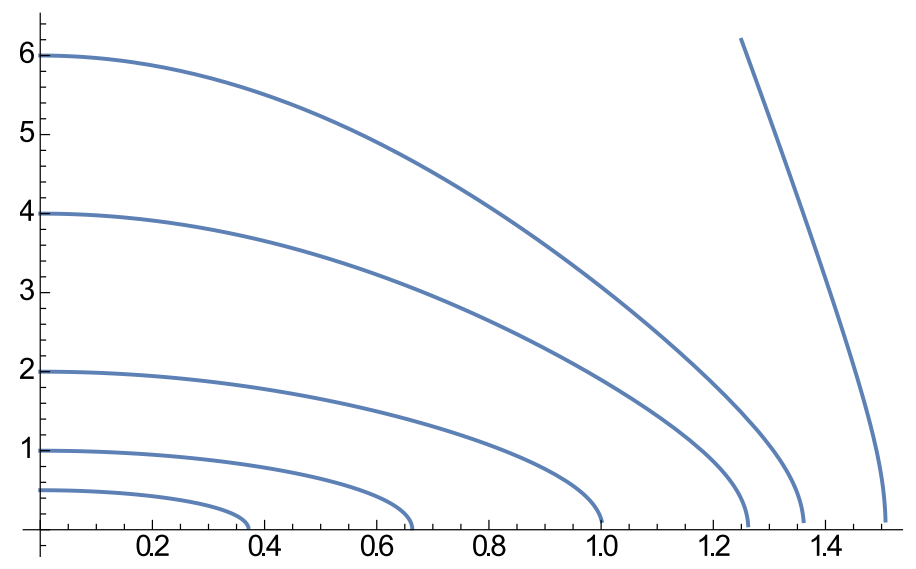

Figure 1. Some examples for the function $h(\vartheta)$, obtained as numerical solutions of the differential equation (2.4) with boundary condition (2.2) and finite $h(0)$.

solved for generic Cauchy data. Furthermore, there appear movable singularities, whose positions depend on the initial conditions. In particular, any zero of $h$ in $(0, \pi / 2)$ can occur only in combination with diverging derivatives.

The solutions regular at $\vartheta=0$ have a power series expansion in $\vartheta^{2}$

$$
h(\vartheta)=h_{0}-\frac{3+2 h_{0}^{2}}{4 h_{0}} \vartheta^{2}+\frac{16 h_{0}^{6}-32 h_{0}^{4}-174 h_{0}^{2}-135}{384 h_{0}^{3}\left(1+h_{0}^{2}\right)} \vartheta^{4}+\mathcal{O}\left(\vartheta^{6}\right) .
$$

Some examples of numerical solutions are shown in figure 1. Obviously, $h(\vartheta)$ has its maximum value $h_{0}$ at $\vartheta=0$ and falls monotonically to zero at $\vartheta=\Omega$. The relation between $h_{0}$ and $\Omega$ is one to one, the function $h_{0}(\Omega)$ is monotonically increasing.

For our further analysis it is sufficient to solve (2.4) with boundary condition (2.2) near $\vartheta=\Omega$. We get

$$
\begin{aligned}
h(\Omega-\delta)= & 2(\tan \Omega)^{1 / 2} \delta^{1 / 2}+\frac{(3-\cos (2 \Omega))(\cot \Omega)^{1 / 2}}{8 \cos ^{2} \Omega} \delta^{3 / 2} \log \delta \\
& +h_{*} \delta^{3 / 2}+\mathcal{O}\left(\delta^{5 / 2} \log ^{2} \delta\right) .
\end{aligned}
$$

If one looks for arbitrary solutions of (2.4) approaching $h=0$, the constant $h_{*}$ remains free. ${ }^{4}$ For those solutions, depicted in figure 1 , which start with $h(0)=h_{0}$ and smoothly fall down to $h=0$, the constant $h_{*}$ is a function of $h_{0}$. Note also, that due to (2.6) real solutions can approach $h=0$ at $\vartheta=\Omega \in(0, \pi / 2)$ only from the side of lower $\vartheta$-values.

With (2.6) we can confirm a formula taken out of [6]

$$
\sin \vartheta=\sin \Omega-\frac{\cos \Omega \cot \Omega}{4} h^{2}+\frac{(3-\cos (2 \Omega)) \cot ^{2} \Omega}{64 \sin \Omega} h^{4} \log h+\mathcal{O}\left(h^{4}\right) .
$$

Since $h(\vartheta)$ is monotonically decreasing, we can use the inversion $\vartheta(h)$ to change the integration variable in (2.3) from $\vartheta$ to $h$. Defining

$$
F(h)=\frac{\sin \vartheta(h)}{h^{3} \dot{h}(\vartheta(h))} \sqrt{1+h^{2}+\dot{h}^{2}}
$$

\footnotetext{
${ }^{4}$ We have checked explicitly, that in the asymptotic series for the solution of (2.4) the coefficients of the next order terms $\delta^{5 / 2} \log ^{n} \delta, n=0,1,2$ can be expressed in terms of $\Omega$ and $h_{*}$.
} 
one gets $[6]$

$$
F(h)=-\frac{\sin \Omega}{h^{3}}+\frac{\cos \Omega \cot \Omega}{8 h}+\mathcal{O}(h) .
$$

Following the standard UV-regularisation by cutting off that part of the manifold whose distance in the Poincaré coordinate $r$ is smaller than $\epsilon$ and using the IR-cut-off $\rho<l$ we get for the regularised volume of $(2.1)$

$$
V_{\epsilon, l}=2 \pi \int_{h_{0}}^{\epsilon / l} d h F(h) \int_{\epsilon / h}^{l} \frac{d \rho}{\rho} .
$$

To handle the divergence of $F(h)$ at $h \rightarrow 0$ we define its finite piece $\tilde{F}$ by

$$
F(h)=\tilde{F}(h)-\frac{\sin \Omega}{h^{3}}+\frac{\cos \Omega \cot \Omega}{8 h} .
$$

Then from $(2.9)$ we know $\tilde{F}=\mathcal{O}(h)$.

Performing the $\rho$-integration one gets

$$
V_{\epsilon, l}=2 \pi \int_{\epsilon / l}^{h_{0}} d h\left(\tilde{F}(h)-\frac{\sin \Omega}{h^{3}}+\frac{\cos \Omega \cot \Omega}{8 h}\right) \log \frac{\epsilon}{l h} .
$$

In the limit $\epsilon \rightarrow 0$ in the part of the integral with $\tilde{F}$ one can replace the lower boundary by zero. The remaining parts of the $h$-integral can be performed explicitly. The final result is

$$
\begin{aligned}
V_{\epsilon, l}= & \frac{\pi \sin \Omega}{2} \frac{l^{2}}{\epsilon^{2}}-\frac{\pi \cos \Omega \cot \Omega}{8} \log ^{2} \frac{\epsilon}{l}+o(1) \\
& +\left(2 \pi \int_{0}^{h_{0}} \tilde{F}(h) d h+\frac{\pi \sin \Omega}{h_{0}^{2}}+\frac{\pi \cos \Omega \cot \Omega}{4} \log h_{0}\right) \log \frac{\epsilon}{l} \\
& -2 \pi \int_{0}^{h_{0}} d h \tilde{F} \log h-\frac{\pi\left(1+2 \log h_{0}\right) \sin \Omega}{2 h_{0}^{2}}-\frac{\pi \cos \Omega \cot \Omega}{8} \log ^{2} h_{0} .
\end{aligned}
$$

The coefficient of the most singular term $1 / \epsilon^{2}$ is equal to half of the area of the boundary of the cone cut at $\rho=l$.

Some further observations on the stability issue of these solutions, which go beyond the discussion in [6], are presented in the next section.

\section{Stability analysis for the cone solutions and study of hollow cone solutions}

Depending on the initial data, the numerical evaluation of (2.4) stops at some points $(\vartheta, h)$ with $\vartheta, h \geq 0$ where $\dot{h}(\vartheta) \rightarrow \pm \infty$, see figure 2 . We expect that this is an artefact of the chosen parameterisation of the minimal submanifold. It continues smoothly beyond such a point, just in a manner that the inverse function $\vartheta(h)$ has a local minimum. To prove this statement, we transform the differential equation (2.4) in one for the inverse function and get (' denoting $d / d h)$

$$
\vartheta^{\prime \prime}=\frac{1+\vartheta^{\prime 2}\left(1+h^{2}\right)}{1+h^{2}} \cot \vartheta(h)+\frac{\vartheta^{\prime}}{h} \frac{3+h^{2}+\vartheta^{\prime 2}\left(3+5 h^{2}+2 h^{4}\right)}{1+h^{2}} .
$$




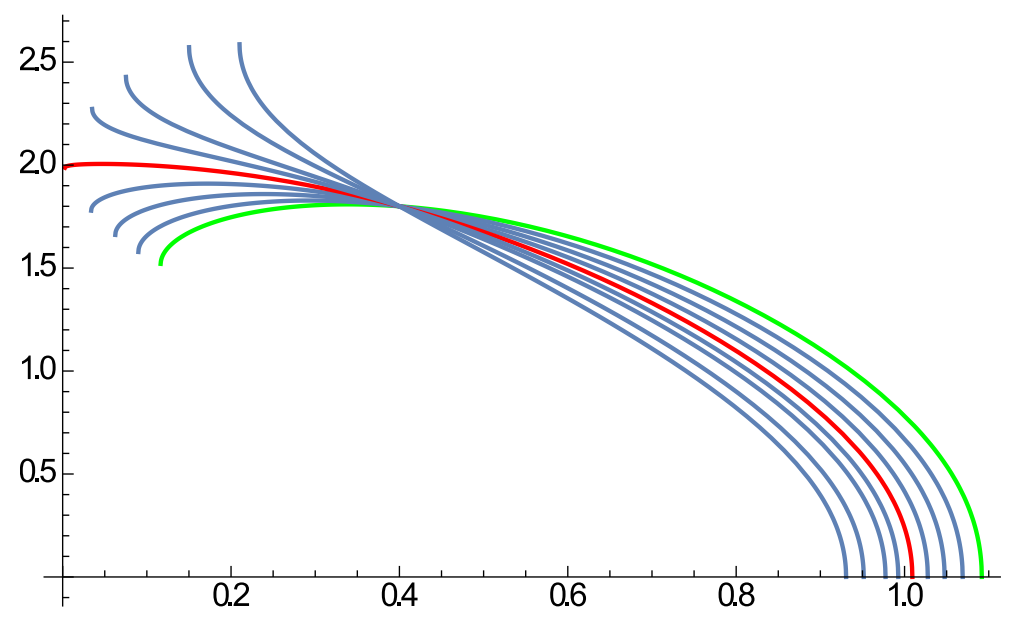

Figure 2. Numerical solutions $h(\vartheta)$ of the differential equation (2.4), obtained by solving the Cauchy initial problem at $\vartheta=0.4$ with $h=1.8$ and different values for $\dot{h}$. The red curve in the middle corresponds to an approximation for a solution reaching its maximum $h_{0}$ at $\vartheta=0$. The green marking is made for reference to figure 3 .

Obviously, points with $h \neq 0$ and $0<\vartheta<\pi$ are regular, allowing solutions of the full Cauchy initial value problem. With $\vartheta^{\prime}=0$ (corresponding to $\dot{h}= \pm \infty$ ) one arrives at

$$
\vartheta^{\prime \prime}=\frac{\cot \vartheta}{1+h^{2}},
$$

showing that for the function $\vartheta(h)$ local extrema can appear in the strip $0<\vartheta<\pi / 2$ ( $h=0$ still excluded) as minima only and in the strip $\pi / 2<\vartheta<\pi$ as maxima only. In a similar way one gets from the original differential equation (2.4), that in $0<\vartheta<\pi$ at local extrema $\ddot{h} / h<0$, excluding therefore local minima of $h(\vartheta)$ for $h>0$ and local maxima for $h<0$. This gives us already a lot of information on the smooth continuations of the curves in figure 2. Concerning the vertical direction only turns into the direction of the $\vartheta$-axis are allowed. In horizontal direction only turns into the direction of the line $\vartheta=\pi / 2$ can show up.

To illustrate the situation by a typical example, we have fine-tuned the numerical evaluation of the Cauchy initial problem for (2.4) at a point closely located near the upper endpoint of the green curve in figure 2. The result is depicted in figure 3 . It shows that the smooth continuations of the curves in figure 2 give us solutions to the boundary problem

$$
h\left(\Omega_{1}\right)=h\left(\Omega_{2}\right)=0, \quad \text { with } \Omega_{1}<\Omega_{2} .
$$

With such boundary conditions the volume of our 3-dimensional minimal submanifold (2.1) via (1.2) is a candidate for the entanglement entropy of special hollow cone regions whose boundaries are given by the surfaces of two cones with common axis and common tip, but different opening angles $\Omega_{1}<\Omega_{2}$. For cones one needs only $\Omega \in(0, \pi / 2)$. To cover all possible interesting cases of hollow cones, one should keep in mind $\Omega_{1} \in(0, \pi / 2)$ and $\Omega_{2} \in(0, \pi)$.

The continuation for curves closer and closer to the original red one in figure 2 yields hollow cone solutions with smaller and smaller $\Omega_{2}-\Omega_{1}$. This shows a remarkable instability 

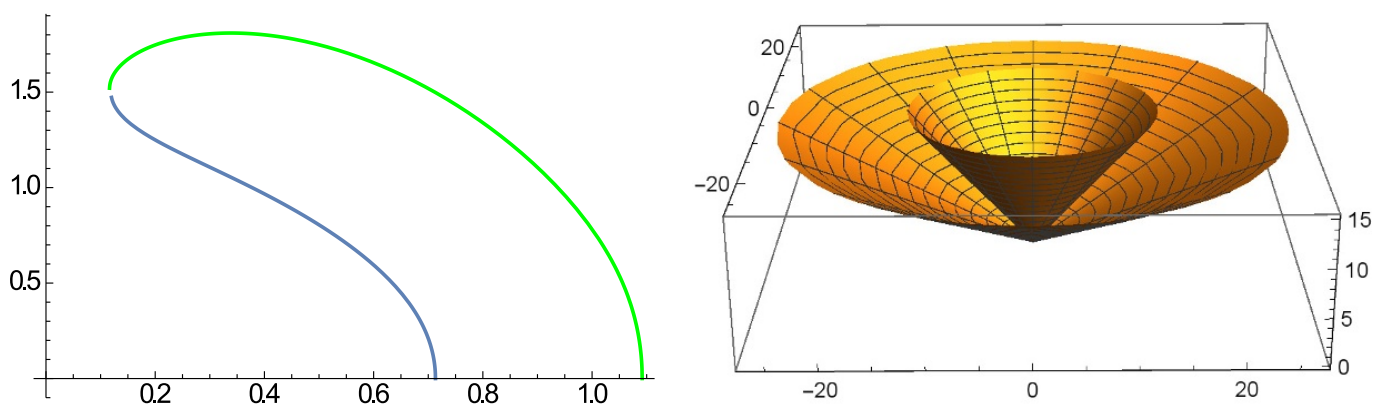

Figure 3. On the left: numerical solution of the diff. equation (2.4), obtained by fitting a second piece to the green curve in figure 2. On the right: the inner and outer boundary of the related hollow cone.

of the cone solutions. As soon as one acts on a cone solution with an arbitrary small perturbation away from fine-tuned Cauchy data, it immediately jumps to a hollow cone solution with $\Omega_{1}$ very close to $\Omega_{2} \cdot{ }^{5}$ To stay smoothly within the set of cone solutions, one has to restrict oneself to only fine-tuned variations of already fine-tuned Cauchy data.

On the other side, the situation can also be seen as a smooth one. The turns of the hollow cone curves in close neighbourhood of the $h$-axis are some kind of smooth reflections, and in the limit $\Omega_{1} \rightarrow \Omega_{2}$ one reaches hard reflection, where one goes back on the infalling curve.

As a side remark we comment on the situation in the lower dimensional case, which has been mentioned in the introduction. There the equation for the analog of $h$ allows generic Cauchy data for all $h>0$. A small variation away from a certain choice of Cauchy data, which guarantee single cusp boundary conditions, results in another cusp situation with slightly varied cusp angle. Hence under variation of Cauchy data these solutions are stable within the class of boundary conditions for a single cusp.

Let us now turn to the evaluation of the volume of the AdS manifold (2.1), with $h(\vartheta)$ obeying the hollow cone boundary conditions (3.3). At first we have to handle the fact, that now, different from the cone case, the relation between $\vartheta$ and $h$ is no longer one to one. Introducing some parameter $t$ on the curve in the $(\vartheta, h)$-plot, we define

$$
h_{0}=\max _{t} h(t), \quad \vartheta_{0}=\min _{t} \vartheta(t) .
$$

Both $h_{0}$ and $\vartheta_{0}$ are functions of $\Omega_{1}$ and $\Omega_{2}$.

Denoting by $h_{1}(\vartheta) \leq h_{2}(\vartheta)$ the two pieces of the curve $(\vartheta(t), h(t)),{ }^{6}$ the unregularised expression for the volume of the AdS submanifold (2.1) with boundary condition (3.3) becomes

$$
V^{\text {h.c. }}=2 \pi \int_{0}^{\infty} \frac{d \rho}{\rho}\left(\int_{\vartheta_{0}}^{\Omega_{1}} d \vartheta \frac{\sin \vartheta}{h_{1}^{3}(\vartheta)} \sqrt{1+h_{1}^{2}+\dot{h}_{1}^{2}}+\int_{\vartheta_{0}}^{\Omega_{2}} d \vartheta \frac{\sin \vartheta}{h_{2}^{3}(\vartheta)} \sqrt{1+h_{2}^{2}+\dot{h}_{2}^{2}}\right) .
$$

\footnotetext{
${ }^{5}$ As discussed at the end of this section, for such very thin hollow cones these solutions are not the one with smallest volume and therefore not responsible for the hollow cone entropy.

${ }^{6}$ The blue and green part in the example of figure 3.
} 
For the separation of the divergences it is more convenient to proceed along the lines of the previous section, changing the integration variable from $\vartheta$ to $h$.

On the piece of the curve, described by $h_{1}(\vartheta)$, the relation between $\vartheta$ and $h$ is one to one, and we can define $F_{1}(h)$ similar to $(2.8)$ by

$$
F_{1}(h)=\frac{\sin \vartheta_{1}(h)}{h^{3} \dot{h}_{1}\left(\vartheta_{1}(h)\right)} \sqrt{1+h^{2}+\dot{h}_{1}^{2}}
$$

On the other piece, described by $h_{2}(\vartheta)$, one has to choose on the parts left and right of the maximum of $h_{2}$ the appropriate branch of the (not unique) inversion of the function $h_{2}(\vartheta)$. With this in mind we define for $\vartheta \in\left(\vartheta\left(h_{0}\right), \Omega_{2}\right)$

$$
F_{2}(h)=\frac{\sin \vartheta_{2}^{r}(h)}{h^{3} \dot{h}_{2}\left(\vartheta_{2}^{r}(h)\right)} \sqrt{1+h^{2}+\dot{h}_{2}^{2}}, \quad h_{2}\left(\vartheta_{2}^{r}(h)\right)=h, \quad \frac{d \vartheta_{2}^{r}(h)}{d h}<0,
$$

as well as for $\vartheta \in\left(\vartheta_{0}, \vartheta\left(h_{0}\right)\right)$

$$
\hat{F}_{2}(h)=\frac{\sin \vartheta_{2}^{l}(h)}{h^{3} \dot{h}_{2}\left(\vartheta_{2}^{l}(h)\right)} \sqrt{1+h^{2}+\dot{h}_{2}^{2}}, \quad h_{2}\left(\vartheta_{2}^{l}(h)\right)=h, \quad \frac{d \vartheta_{2}^{l}(h)}{d h}>0 .
$$

The regularised volume is then

$$
\begin{aligned}
V_{\epsilon, l}^{\text {h.c. }}= & 2 \pi \int_{h\left(\vartheta_{0}\right)}^{\epsilon / l} d h F_{1}(h) \int_{\epsilon / h}^{l} \frac{d \rho}{\rho}+2 \pi \int_{h_{0}}^{\epsilon / l} d h F_{2}(h) \int_{\epsilon / h}^{l} \frac{d \rho}{\rho} \\
& +2 \pi \int_{h\left(\vartheta_{0}\right)}^{h_{0}} d h \hat{F}_{2}(h) \int_{\epsilon / h}^{l} \frac{d \rho}{\rho} .
\end{aligned}
$$

One has $F_{2}(h) \leq 0$ and $\hat{F}_{2}(h) \geq 0$. Near $h_{0}$ they both become singular like $\mp\left(h_{0}-h\right)^{-1 / 2}$. Since this singularity is integrable, the third term in (3.9) contributes to the divergent piece of $V_{\epsilon, l}^{\text {h.c. }}$ only via the divergence of the $\rho$-integration. The divergences arising from the first two terms are due to divergence of the $\rho$-integration and the behaviour of $F_{1}$ and $F_{2}$ at $h=0$. This on its part is determined by $h_{1}$ and $h_{2}$ near $\vartheta=\Omega_{1}$ and $\vartheta=\Omega_{2}$, respectively. Since both functions are solutions of (2.4) we can apply (2.6). The corresponding values for the constant $h_{*}$ are now tuned differently from the construction in the previous section. But since anyway its value was not relevant for the evaluation of the divergent parts we get

$$
\begin{aligned}
V_{\epsilon, l}^{\text {h.c. }}= & \frac{\pi}{2}\left(\sin \Omega_{1}+\sin \Omega_{2}\right) \frac{l^{2}}{\epsilon^{2}}-\frac{\pi}{8}\left(\cos \Omega_{1} \cot \Omega_{1}+\cos \Omega_{2} \cot \Omega_{2}\right) \log ^{2} \frac{\epsilon}{l} \\
& +\left(2 \pi \int_{0}^{h\left(\vartheta_{0}\right)} \tilde{F}_{1}(h) d h+2 \pi \int_{0}^{h_{0}} \tilde{F}_{2}(h) d h+2 \pi \int_{h_{0}}^{h\left(\vartheta_{0}\right)} \hat{F}_{2}(h) d h\right. \\
& +\frac{\pi \sin \Omega_{1}}{\left(h\left(\vartheta_{0}\right)\right)^{2}}+\frac{\pi \cos \Omega_{1} \cot \Omega_{1}}{4} \log h\left(\vartheta_{0}\right) \\
& \left.+\frac{\pi \sin \Omega_{2}}{h_{0}^{2}}+\frac{\pi \cos \Omega_{2} \cot \Omega_{2}}{4} \log h_{0}\right) \log \frac{\epsilon}{l}+\mathcal{O}(1)
\end{aligned}
$$

$\tilde{F}_{1}$ and $\tilde{F}_{2}$ are defined according to (2.11) with the corresponding index. 


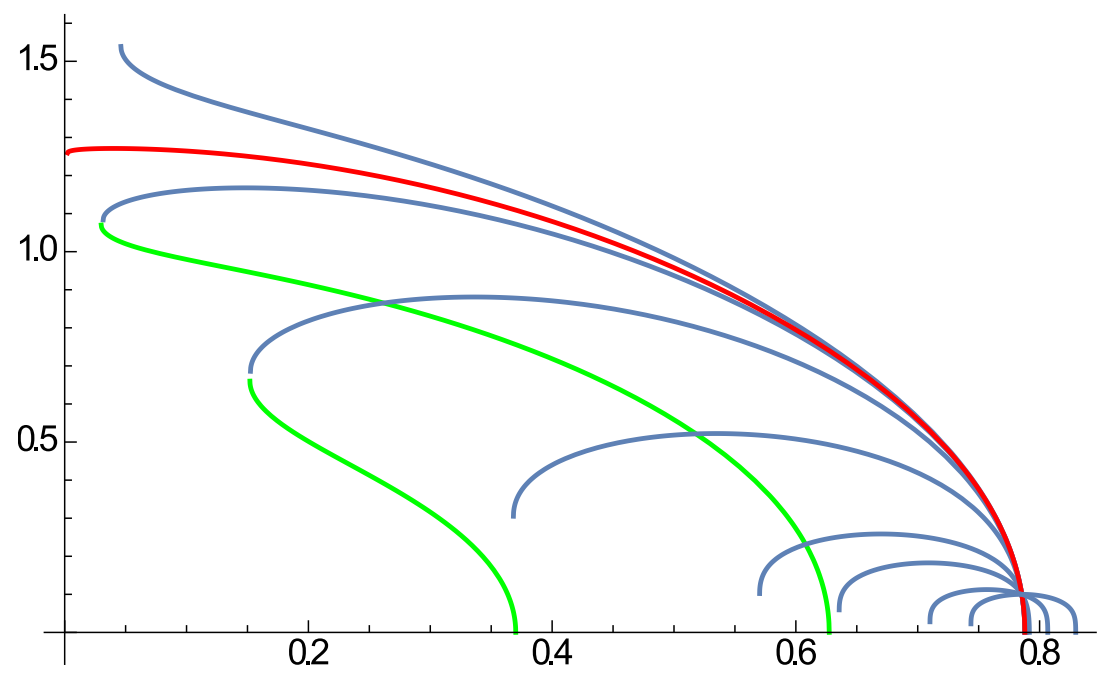

Figure 4. Numerical solutions $h(\vartheta)$ of $(2.4)$ with $h(\pi / 4)=0.1$ and $\dot{h}(\pi / 4) \in(0,-1,-5,-10,-18$, $-19.5,-19.7,-19.724,-19.75)$, plotted for $h>0$ and down to values of $\vartheta$, where $\dot{h}$ diverges (in blue, in red for the approximate cone solution). For two of these curves approximate smooth continuations are shown in green.

The leading and the nextleading divergent terms are just the sum of the corresponding terms for the two single cones of opening angles $\Omega_{1}$ and $\Omega_{2}$. For the leading term this is due to the additivity of the area of $\partial \mathcal{A}$. For the coefficient of the $\log ^{2} \epsilon$, which is due to the singularities of $\partial \mathcal{A}$, it could have been expected since the complement of a hollow cone consists of two full cones. Then our calculation shows that touching each other at their tips does not disturbe additivity for the leading and nextleading terms.

The difference from simple additivity begins with the $\log \frac{\epsilon}{l}$ term. Note that $\vartheta_{0}$ and $h_{0}$ are both functions of $\Omega_{1}$ and $\Omega_{2}$.

It is interesting to compare this situation with that for the entanglement entropy of two regions with just one common point in a lower dimensional setting [14]. In both cases deviations from additivity appear just for the $\log \frac{\epsilon}{l}$ term. However, one has to keep in mind that $\log \frac{\epsilon}{l}$ terms for $d=3$ appear also in the smooth case, while for $d=2$ they are special for regions with singularities in their boundary.

Of course the disconnected 3-dimensional manifold consisting out of the manifolds for the two single cones ${ }^{7}$ always fulfils also our differential equation (2.4) and the hollow cone boundary conditions (3.3). The decision for which angles $\Omega_{1}, \Omega_{2}$ the connected version, by its smaller volume, is favoured with respect to the disconnected version needs involved numerical analysis of the $\log \frac{\epsilon}{l}$ term of both candidates.

However, one gets a clear qualitative picture by plotting solutions obtained from Cauchy data at a point very close to the $\vartheta$-axis, see figure 4 . For small initial values of $|\dot{h}|$ the curve stays in the vicinity of the initial point. Choosing negative $\dot{h}$, but larger $|\dot{h}|$, the curves get larger maxima $h_{0}$, their minima in $\vartheta$, i.e. $\vartheta_{0}$, move further down on the

\footnotetext{
${ }^{7}$ To be precise: disconnected inside AdS, the two pieces touch each other on the boundary of AdS at the common tip of the cones.
} 

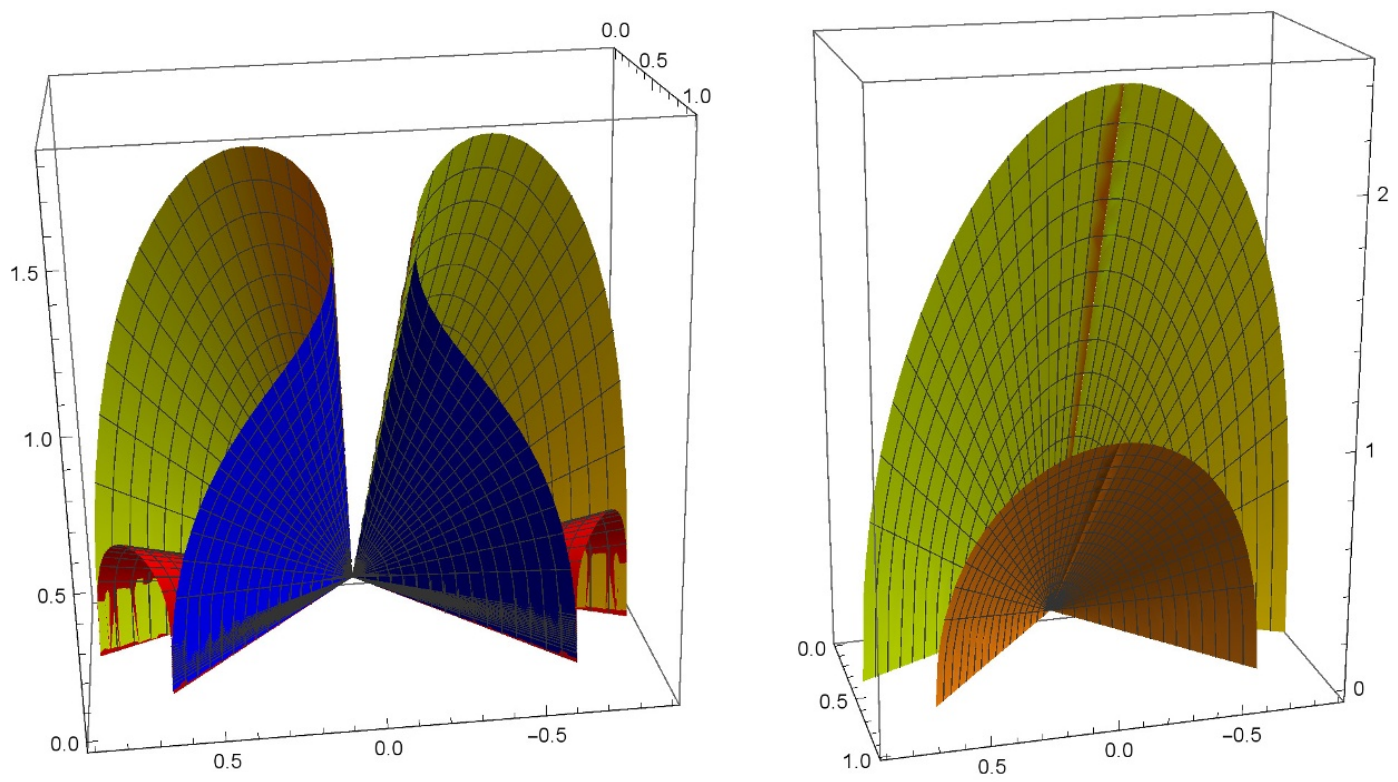

Figure 5. The bottom is the subspace $x_{2}=0$ of the boundary of $A d S_{4}$. The upward direction points inside AdS. On the left: the two connected solutions. On the right: the disconnected solution. The corresponding hollow cone is that from figure 3 .

$\vartheta$-axis and the angular thickness of the related hollow cone, the difference $\Omega_{2}-\Omega_{1}$, becomes larger. One can continue until $\vartheta_{0}$ approaches zero. But remarkably, somewhere in between the angular thickness $\Omega_{2}-\Omega_{1}$ no longer increases and goes back to zero instead.

From this observation we learn the following lessons. To each outer angle $\Omega_{2}$ belongs a limiting value $\Omega_{\min }\left(\Omega_{2}\right)$ for $\Omega_{1}$. For $\Omega_{\min }\left(\Omega_{2}\right)<\Omega_{1}<\Omega_{2}$ one gets two connected solutions. For hollow cones with very small $\Omega_{2}-\Omega_{1}$ the one with smaller $h_{0}$ has smaller volume. This can be seen as following. The coefficients of the two leading divergences in (3.10) are the same for both solutions. The coefficient of the logarithmic divergence stands in front of a negative term and goes to plus infinity for $h_{0}, h\left(\vartheta_{0}\right) \rightarrow 0$. Obviously, by an analogous reasoning, the volume of this favoured connected solution is also smaller than that of the corresponding disconnected solution.

Furthermore, there exists no connected solution if $0<\Omega_{1}<\Omega_{\min }\left(\Omega_{2}\right)$. Hence in varying $\Omega_{1}$ from values near $\Omega_{2}$ to such below $\Omega_{\min }\left(\Omega_{2}\right)$, there has to be a transition of the related entanglement entropy from connected to disconnected solutions. Whether it happens at once from the connected solution with the smaller $h_{0}$ to the disconnected solution, or whether there appears first a transition between the two connected solutions, has to be left open for further study. These should also clarify the order of that geometrically induced phase transition pattern.

We close this section with a visualisation of the extension of the connected and disconnected hollow cone solutions into the interior of AdS. Figure 5 shows the intersection of the 3-dimensional submanifolds corresponding to the hollow cone of figure 3 with the codimension one subspace $x_{2}=0$. One should not be confused by the fact that the projections 
shown in the left part look disconnected, the full 3-dimensional manifolds are connected via the suppressed $x_{2}$-direction.

\section{Elementary geometry of banana shaped regions}

As an example for a compact region, whose boundary has two conical singularities, we take the banana shaped region obtained by a suitable conformal map of the infinite cone. We apply the inversion at the unit sphere

$$
x_{\mu} \mapsto \frac{x_{\mu}}{x^{2}}
$$

to an infinite cone of opening angle $2 \Omega$ with its tip located at $x_{\mu}=(0,0, q)$ and its axis being situated in the $\left(x_{1}, x_{3}\right)$-plane with an angle $\alpha$ relative to the $x_{3}$-axis. Obviously it is sufficient to restrict both $\Omega$ and $\alpha$ to the interval $(0, \pi / 2)$.

This procedure gives the region, see figure 6 ,

$$
\begin{aligned}
& x_{1}(\rho, \vartheta, \varphi)=\frac{\rho \cos \alpha \sin \vartheta \cos \varphi+\rho \sin \alpha \cos \vartheta}{q^{2}+\rho^{2}+2 q \rho(\cos \alpha \cos \vartheta-\sin \alpha \sin \vartheta \cos \varphi)}, \\
& x_{2}(\rho, \vartheta, \varphi)=\frac{\rho \sin \vartheta \sin \varphi}{q^{2}+\rho^{2}+2 q \rho(\cos \alpha \cos \vartheta-\sin \alpha \sin \vartheta \cos \varphi)}, \\
& x_{3}(\rho, \vartheta, \varphi)=\frac{q+\rho \cos \alpha \cos \vartheta-\rho \sin \alpha \sin \vartheta \cos \varphi}{q^{2}+\rho^{2}+2 q \rho(\cos \alpha \cos \vartheta-\sin \alpha \sin \vartheta \cos \varphi)} .
\end{aligned}
$$

The coordinates $\rho, \vartheta$ and $\varphi$ obey

$$
0 \leq \rho<\infty, \quad 0 \leq \vartheta \leq \Omega, \quad 0 \leq \varphi<2 \pi .
$$

The transformation (4.1) preserves angles and maps spheres to spheres and circles to circles (planes and straight lines understood as spheres and circles passing infinity).

Therefore, the boundary surface of the region (4.2) has two conical singularities of opening angle $2 \Omega$, whose distance $D$ is

$$
D=\frac{1}{q}
$$

and which are located at $(0,0,1 / q)$ and at the origin. This boundary surface is described by $x_{\mu}(\rho, \Omega, \varphi)$, with $\rho$ and $\varphi$ as coordinates. The lines of constant $\varphi$ are segments of circles passing the two singular points and having the radii

$$
R_{\alpha, \Omega, q}(\varphi)=\frac{1}{2 q \sqrt{1-(\cos \alpha \cos \Omega-\sin \alpha \sin \Omega \cos \varphi)^{2}}} .
$$

The minimal and maximal values of these radii are

$$
R_{\alpha, \Omega, q}^{\min / \max }=\frac{1}{2 q|\sin (\alpha \pm \Omega)|} .
$$

The axis of the banana (4.2) is a segment of a circle of radius

$$
R_{\alpha, \Omega, q}^{\mathrm{axis}}=\frac{1}{2 q \sin \alpha} .
$$



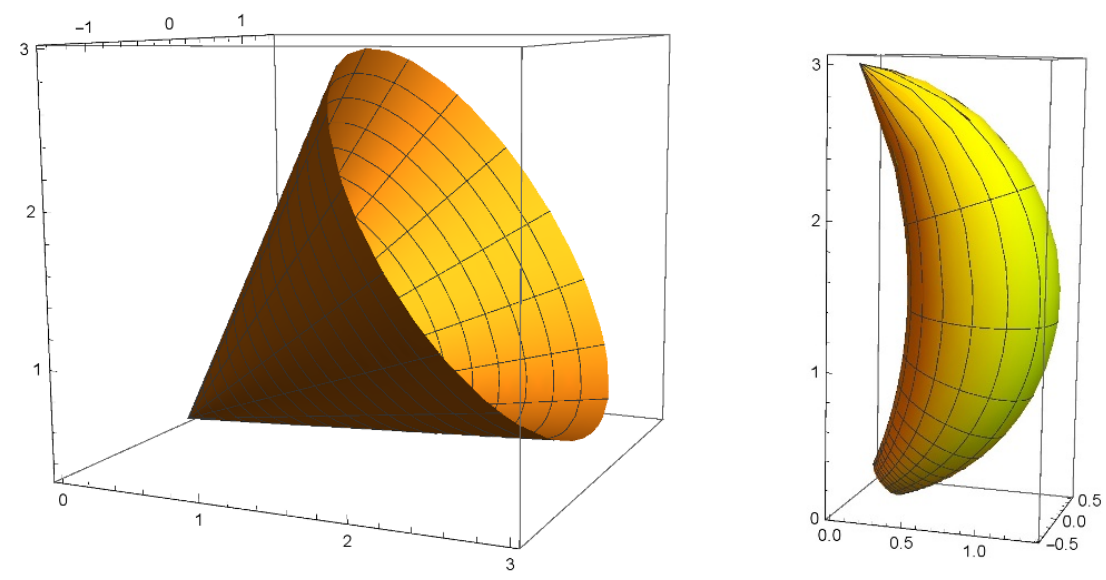

Figure 6. The map (4.1) for $q=1 / 3, \alpha=1, \Omega=0.5$. Both for the preimage and the image we show $0<\rho<3$, only.

Furthermore, the lines of constant $\rho$ are complete circles. They are intersections of the boundary surface with two-spheres, touching one of the singular points and having their center in the plane dividing the banana into two halves. Among those circles the largest one has a radius

$$
R_{\alpha, \Omega, q}^{\perp}=\frac{\sin \Omega}{2 q(\cos \alpha+\cos \Omega)} .
$$

The metrical geometry of the boundary surface of our banana shaped region (4.2) is fixed by the dimensionful parameter $q$ and the two angles $\alpha$ and $\Omega$. Using (4.4), (4.6)-(4.8) these parameters can be expressed in terms of three out of the parameters measuring length distances related to the boundary surface.

For later use we are still interested in the area of the boundary surface. The square root of the induced metrics determinant is

$$
\sqrt{g}=\frac{\rho \sin \Omega}{\left(q^{2}+\rho^{2}+2 q \rho(\cos \alpha \cos \Omega-\sin \alpha \sin \Omega \cos \varphi)\right)^{2}}
$$

Performing either the $\varphi$-integration or the $\rho$-integration we arrive at ${ }^{8}$

$$
\begin{aligned}
A_{\alpha, \Omega, q} & =\frac{2 \pi \sin \Omega}{q^{2}} \int_{0}^{\infty} \frac{x\left(1+x^{2}+2 x \cos \alpha \cos \Omega\right) d x}{\left(\left(1+x^{2}+2 x \cos (\alpha+\Omega)\right)\left(1+x^{2}+2 x \cos (\alpha-\Omega)\right)\right)^{3 / 2}}, \\
& =\frac{\sin \Omega}{2 q^{2}}\left(\frac{\pi}{\cos \alpha+\cos \Omega}+\frac{\pi}{|\cos \alpha-\cos \Omega|}-\int_{0}^{2 \pi} \frac{\hat{w}(\varphi) \arccos \hat{w}}{\left(1-\hat{w}^{2}\right)^{3 / 2}} d \varphi\right) .
\end{aligned}
$$

\footnotetext{
${ }^{8}$ The remaining integrals can be expressed in terms of elliptic functions, but we did not find a short compact expression.
} 
In the second line also part of the $\varphi$-integration has been performed using the integral

$$
\int_{0}^{2 \pi} \frac{d \varphi}{1-\hat{w}^{2}(\varphi)}=\frac{\pi}{\cos \alpha+\cos \Omega}+\frac{\pi}{|\cos \alpha-\cos \Omega|}
$$

where

$$
\hat{w}(\varphi)=\cos \alpha \cos \Omega-\sin \alpha \sin \Omega \cos \varphi .
$$

Some special cases of (4.10) are

$$
\begin{aligned}
A_{\Omega, \Omega, q} & =\frac{\pi}{16 q^{2}} \frac{2 \sin \Omega+2 \sin ^{3} \Omega-\cos ^{4} \Omega \log \left(\frac{1+\sin \Omega}{1-\sin \Omega}\right)}{\sin ^{2} \Omega \cos ^{2} \Omega}, \\
A_{0, \Omega, q} & =\frac{\pi}{q^{2} \sin \Omega}(1-\Omega \cot \Omega), \\
A_{\alpha, \pi / 2, q} & =\frac{\pi}{q^{2} \cos ^{2} \alpha} .
\end{aligned}
$$

\section{$5 \quad$ Entanglement entropy for banana shaped regions}

As in the previous section we shift the tip of the cone in the boundary of AdS out of the origin and tilt its axis relative to the $x_{3}$-axis. Then the corresponding 3 -manifold in $A d S_{4}$ is

$$
\begin{aligned}
x_{1} & =\rho \cos \alpha \sin \vartheta \cos \varphi+\rho \sin \alpha \cos \vartheta, \\
x_{2} & =\rho \sin \vartheta \sin \varphi \\
x_{3} & =q+\rho \cos \alpha \cos \vartheta-\rho \sin \alpha \sin \vartheta \cos \varphi, \\
r & =\rho h(\vartheta),
\end{aligned}
$$

with $h(\vartheta)$ the same function as in section 2.

Now we apply the mapping

$$
x_{\mu} \mapsto \frac{x_{\mu}}{x^{2}+r^{2}}, \quad r \mapsto \frac{r}{x^{2}+r^{2}},
$$

which is an AdS-isometry and acts conformally on the boundary $r=0$. Then we get the submanifold of our interest

$$
\begin{aligned}
x_{1} & =\frac{\rho(\cos \alpha \sin \vartheta \cos \varphi+\sin \alpha \cos \vartheta)}{q^{2}+\rho^{2}\left(1+h^{2}(\vartheta)\right)+2 q \rho w(\vartheta, \varphi)}, \\
x_{2} & =\frac{\rho \sin \vartheta \sin \varphi}{q^{2}+\rho^{2}\left(1+h^{2}(\vartheta)\right)+2 q \rho w(\vartheta, \varphi)}, \\
x_{3} & =\frac{q+\rho w(\alpha, \vartheta, \varphi)}{q^{2}+\rho^{2}\left(1+h^{2}(\vartheta)\right)+2 q \rho w(\vartheta, \varphi)}, \\
r & =\frac{\rho h(\vartheta)}{q^{2}+\rho^{2}\left(1+h^{2}(\vartheta)\right)+2 q \rho w(\vartheta, \varphi)},
\end{aligned}
$$

with

$$
w(\vartheta, \varphi)=\cos \alpha \cos \vartheta-\sin \alpha \sin \vartheta \cos \varphi .
$$

Here $\alpha$ and $q$ are parameters specifying the submanifold, and $\rho, \vartheta, \varphi$ are coordinates. 
Since the map (5.2) is an isometry, the volume of the manifold (5.3) is given by an integral over the coordinates with the same integrand as in section 2. But now there is no need for an IR-regularisation and the cut-off for the UV-regularisation introduces modified boundaries for the coordinates.

The condition $r=\epsilon$ has two solutions for $\rho$ as a function of $h$ and $\varphi$

$$
\rho_{\epsilon}^{( \pm)}(h, \varphi)=\frac{h-2 q \epsilon w(\vartheta(h), \varphi) \pm \sqrt{(h-2 q \epsilon w)^{2}-4 \epsilon^{2} q^{2}\left(1+h^{2}\right)}}{2 \epsilon\left(1+h^{2}\right)} .
$$

Let us introduce for later convenience

$$
N_{\epsilon}(h, \varphi)=1-\frac{2 q \epsilon}{h} w(\vartheta(h), \varphi)+\sqrt{\left(1-\frac{2 q \epsilon w}{h}\right)^{2}-4 \epsilon^{2} q^{2} \frac{1+h^{2}}{h^{2}}} .
$$

Then one has

$$
\rho_{\epsilon}^{(+)}=\frac{h}{2 \epsilon\left(1+h^{2}\right)} \cdot N_{\epsilon}(h, \varphi), \quad \rho_{\epsilon}^{(-)}=\frac{2 q^{2} \epsilon}{h} \cdot \frac{1}{N_{\epsilon}(h, \varphi)},
$$

and the interval for the $\rho$-integration will be

$$
\rho_{\epsilon}^{(-)}<\rho<\rho_{\epsilon}^{(+)}
$$

The expression under the square root in (5.5) has to be positive. This constrains $h$ from below, i.e.

$$
h_{\epsilon}<h<h_{0},
$$

where $h_{0}$ as a function of $\Omega$ has been defined in section 2 and

$$
h_{\epsilon}(\varphi)=\frac{2 q \epsilon}{1-4 q^{2} \epsilon^{2}}\left(w\left(\vartheta\left(h_{\epsilon}\right), \varphi\right)+\sqrt{1-4 q^{2} \epsilon^{2}+4 q^{2} \epsilon^{2} w^{2}}\right) .
$$

Note that $h_{\epsilon}$ appears also on the r.h.s. as argument of the function $\vartheta(h)$. The reality condition for the square root in (5.5) allows also a minus in front of the square root in (5.10), but the analysis for small $\epsilon$ shows, that one has to choose the positive sign to ensure positive values for $h_{\epsilon}$. With (2.7) we find the expansion

$$
\begin{aligned}
& h_{\epsilon}(\varphi)=B_{1} q \epsilon+B_{3} q^{3} \epsilon^{3}+\ldots, \quad \text { with } \\
& B_{1}(\varphi)=2(1+\hat{w}(\varphi)), \\
& B_{3}(\varphi)=2(1+\hat{w}(\varphi))^{2}(2+\cos \Omega(\cos \alpha+\sin \alpha \cot \Omega \cos \varphi)) .
\end{aligned}
$$

The function $\hat{w}(\varphi)=w(\Omega, \varphi)$ has been defined in (4.12), see also (5.4).

After this discussion of the region of integration, the regularised volume is

$$
V_{\epsilon}=-\int_{0}^{2 \pi} d \varphi \int_{h_{\epsilon}}^{h_{0}} d h \int_{\rho_{\epsilon}^{(-)}}^{\rho_{\epsilon}^{(+)}} \frac{d \rho}{\rho} F(h) .
$$

Performing the trivial $\rho$ integration we get with (5.7)

$$
V_{\epsilon}=V_{\epsilon}^{(1)}+V_{\epsilon}^{(2)}+V_{\epsilon}^{(3)}
$$


where

$$
\begin{aligned}
V_{\epsilon}^{(1)} & =-2 \int_{0}^{2 \pi} d \varphi \int_{h_{\epsilon}(\varphi)}^{h_{0}} d h F(h) \log N_{\epsilon}(h, \varphi), \\
V_{\epsilon}^{(2)} & =2 \log (2 q \epsilon) \int_{0}^{2 \pi} d \varphi \int_{h_{\epsilon}(\varphi)}^{h_{0}} d h F(h), \\
V_{\epsilon}^{(3)} & =\int_{0}^{2 \pi} d \varphi \int_{h_{\epsilon}(\varphi)}^{h_{0}} d h F(h) \log \left(\frac{1+h^{2}}{h^{2}}\right) .
\end{aligned}
$$

The function $F(h)$ has been defined in (2.8). To handle the divergence of $F(h)$ at $h \rightarrow 0$ we use (2.11) with $\tilde{F}=\mathcal{O}(h)$.

Now $V_{\epsilon}^{(1)}$ is the most involved part, since, besides the manifest $h$-dependence of its integrand, there is also one via $\vartheta(h)$ in $N_{\epsilon}$, see (5.6). Therefore let us start with

$$
V_{\epsilon}^{(2)}=2 \log (2 q \epsilon) \int_{0}^{2 \pi} d \varphi \int_{h_{\epsilon}(\varphi)}^{h_{0}} d h\left(\tilde{F}(h)+\frac{\cos \Omega \cot \Omega}{8 h}-\frac{\sin \Omega}{h^{3}}\right) .
$$

Up to terms vanishing for $\epsilon \rightarrow 0$ we can replace in the integral over $\tilde{F}(h)$ the lower boundary by zero. The integrations over $1 / h^{3}$ and $1 / h$ are trivial, and with (5.11) we get altogether

$$
\begin{aligned}
V_{\epsilon}^{(2)}= & -\left(\frac{\log (q \epsilon)}{q^{2} \epsilon^{2}}+\frac{\log 2}{q^{2} \epsilon^{2}}\right) \sin \Omega \int_{0}^{2 \pi} \frac{d \varphi}{B_{1}^{2}}-\frac{\pi}{2} \cos \Omega \cot \Omega \log ^{2}(q \epsilon) \\
& +(\log (q \epsilon)+\log 2)\left(4 \pi \int_{0}^{h_{0}} \tilde{F} d h+2 \sin \Omega\left(\frac{\pi}{h_{0}^{2}}+\int_{0}^{2 \pi} \frac{B_{3}}{B_{1}^{3}} d \varphi\right)\right. \\
& \left.+\cos \Omega \cot \Omega\left(\frac{\pi}{2} \log h_{0}-\frac{1}{4} \int_{0}^{2 \pi} \log B_{1} d \varphi\right)\right) \\
& -\frac{\pi \log 2}{2} \cos \Omega \cos \Omega \log (q \epsilon)+o(1) .
\end{aligned}
$$

A similar treatment yields

$$
\begin{aligned}
V_{\epsilon}^{(3)}= & \frac{\log (q \epsilon)}{q^{2} \epsilon^{2}} \sin \Omega \int_{0}^{2 \pi} \frac{d \varphi}{B_{1}^{2}}+\frac{\sin \Omega}{q^{2} \epsilon^{2}} \int_{0}^{2 \pi}\left(\frac{1}{2 B_{1}^{2}}+\frac{\log B_{1}}{B_{1}^{2}}\right) d \varphi \\
& +\frac{\pi}{4} \cos \Omega \cot \Omega \log ^{2}(q \epsilon) \\
& +\log (q \epsilon)\left(2 \sin \Omega\left(\pi-\int_{0}^{2 \pi} \frac{B_{3}}{B_{1}^{3}} d \varphi\right)+\frac{1}{4} \cos \Omega \cot \Omega \int_{0}^{2 \pi} \log B_{1} d \varphi\right) \\
& +2 \pi \int_{0}^{h_{0}} \tilde{F} \log \frac{1+h^{2}}{h^{2}} d h \\
& +\sin \Omega\left(\int_{0}^{2 \pi}\left(1-2 \frac{B_{3}}{B_{1}^{3}}\right) \log B_{1} d \varphi+\pi \frac{1+h_{0}^{2}}{h_{0}^{2}}\left(\log \frac{1+h_{0}^{2}}{h_{0}^{2}}-1\right)\right) \\
& +\cos \Omega \cot \Omega\left(\frac{1}{8} \int_{0}^{2 \pi} \log ^{2} B_{1} d \varphi-\frac{\pi}{8} \operatorname{Li}_{2}\left(-h_{0}^{2}\right)-\frac{\pi}{4} \log ^{2} h_{0}\right)+o(1) .
\end{aligned}
$$


To start the discussion of the asymptotics of $V_{\epsilon}^{(1)}$ at $\epsilon \rightarrow 0$, we note that at any fixed $0 \leq \varphi \leq 2 \pi, h>0$

$$
N_{\epsilon}(h, \varphi)=2+\mathcal{O}(\epsilon) .
$$

Since $\tilde{F}$ tends to zero linearly in $h$, this estimate can be used even if the lower boundary of the $h$-integration tends to zero. This implies

$$
V_{\epsilon}^{(1)}=-4 \pi \log 2 \int_{0}^{h_{0}} \tilde{F}(h) d h+\sin \Omega J_{1}+\cos \Omega \cot \Omega J_{2}+o(1),
$$

with

$$
J_{1}=2 \int_{0}^{2 \pi} d \varphi \int_{h_{\epsilon}(\varphi)}^{h_{0}} \frac{\log N_{\epsilon}(h, \varphi)}{h^{3}} d h, \quad J_{2}=-\frac{1}{4} \int_{0}^{2 \pi} d \varphi \int_{h_{\epsilon}(\varphi)}^{h_{0}} \frac{\log N_{\epsilon}(h, \varphi)}{h} d h
$$

The estimate of these two integrals is performed in appendix A. Putting (A.3) and the results (A.12), (A.14), (A.16) into (5.21) we get

$$
\begin{aligned}
V_{\epsilon}^{(1)}= & \frac{2 \sin \Omega}{q^{2} \epsilon^{2}} \int_{0}^{2 \pi} d \varphi\left(\frac{1}{8\left(1-\hat{w}^{2}\right)}-\frac{1}{16(1+\hat{w})^{2}}-\frac{\hat{w} \arccos \hat{w}}{8\left(1-\hat{w}^{2}\right)^{3 / 2}}-\frac{\log (1+\hat{w})}{8(1+\hat{w})^{2}}\right) \\
& +\frac{\pi \log 2}{2} \cos \Omega \cot \Omega \log (q \epsilon) \\
& -\sin \Omega\left(4 \pi+\frac{2 \pi \log 2}{h_{0}^{2}}+4 \int_{0}^{2 \pi} d \varphi \int_{0}^{1 / B_{1}} \frac{K(\varphi)}{\sqrt{(1-2 x \hat{w})^{2}-4 x^{2}}} d x\right. \\
& \left.-2 \int_{0}^{2 \pi} d \varphi \frac{B_{3}}{B_{1}^{3}} \log (1+\hat{w}(\varphi))\right) \\
& +\cos \Omega \cot \Omega\left(\frac{\log 2}{4} \int_{0}^{2 \pi} \log B_{1} d \varphi-\frac{\pi \log 2}{2} \log h_{0}\right. \\
& \left.-\frac{1}{4} \int_{0}^{2 \pi} d \varphi \int_{0}^{1 / B_{1}} \log \left(\frac{1}{2}-x \hat{w}+\frac{1}{2} \sqrt{(1-2 x \hat{w})^{2}-4 x^{2}}\right) \frac{d x}{x}\right) \\
& -4 \pi \log 2 \int_{0}^{h_{0}} \tilde{F}(h) d h+o(1) .
\end{aligned}
$$

Remember that $B_{1}, B_{3}$ and $\hat{w}$ as functions of $\varphi$ are defined in (5.12) and (4.12). Furthermore, from (A.8) in appendix A we know

$$
K(\varphi)=\frac{1}{2}\left(\frac{B_{3}}{B_{1}^{2}}-1\right)=\frac{1}{4}(\cos \alpha \cos \Omega+\sin \alpha \cos \Omega \cot \Omega \cos \varphi) .
$$

Now we have to add (5.23), (5.18) and (5.19) and get

$$
V_{\epsilon}=\frac{P_{2}}{q^{2} \epsilon^{2}}+L_{2} \log ^{2}(q \epsilon)+L_{1} \log (q \epsilon)+V_{0}+o(1)
$$

In comparison with the general structure (1.4), mentioned in the introduction, this means $c_{1}=P_{2} / q^{2}, a=L_{1}$ and $c_{3}=V_{0}$. Using also (4.11) and

$$
\int_{0}^{2 \pi} \log B_{1}(\varphi) d \varphi=2 \pi \log ((1+\cos \alpha)(1+\cos \Omega))
$$


the coefficients in (5.25) are given by

$$
\begin{aligned}
& P_{2}=\frac{\sin \Omega}{4}\left(\frac{\pi}{\cos \alpha+\cos \Omega}+\frac{\pi}{|\cos \alpha-\cos \Omega|}-\int_{0}^{2 \pi} \frac{\hat{w}(\varphi) \arccos \hat{w}}{\left(1-\hat{w}^{2}\right)^{3 / 2}} d \varphi\right), \\
& L_{2}=-\frac{\pi}{4} \cos \Omega \cot \Omega, \\
& L_{1}=4 \pi \int_{0}^{h_{0}} \tilde{F}(h) d h+2 \pi\left(1+\frac{1}{h_{0}^{2}}\right) \sin \Omega+\frac{\pi}{2} \cos \Omega \cot \Omega \log h_{0}
\end{aligned}
$$

and the finite part by

$$
\begin{aligned}
V_{0}= & \sin \Omega\left(2 \pi \log ((1+\cos \alpha)(1+\cos \Omega))+\frac{\pi\left(1+h_{0}^{2}\right)}{h_{0}^{2}}\left(\log \frac{1+h_{0}^{2}}{h_{0}^{2}}-1\right)\right. \\
& \left.-4 \pi-4 \int_{0}^{2 \pi} \int_{0}^{1 / B_{1}} \frac{K(\varphi)}{\sqrt{(1-2 x \hat{w}(\varphi))^{2}-4 x^{2}}} d x\right) \\
& +\cos \Omega \cot \Omega\left(\frac{1}{8} \int_{0}^{2 \pi} \log ^{2} B_{1}(\varphi) d \varphi-\frac{\pi}{8} \operatorname{Li}_{2}\left(-h_{0}^{2}\right)-\frac{\pi \log ^{2} h_{0}}{4}\right. \\
& \left.-\frac{1}{4} \int_{0}^{2 \pi} d \varphi \int_{0}^{1 / B_{1}} \log \left(\frac{1}{2}-x \hat{w}(\varphi)+\frac{1}{2} \sqrt{(1-2 x \hat{w})^{2}-4 x^{2}}\right) \frac{d x}{x}\right) \\
& +2 \pi \int_{0}^{h_{0}} \tilde{F}(h) \log \frac{1+h^{2}}{h^{2}} d h .
\end{aligned}
$$

For clarity let us repeat the meaning of the entries in these formulae. $\Omega, \alpha, q$ are geometrical parameters of the banana shaped region. In detail, $2 \Omega$ is the opening angle of its tips, $\alpha$ is the angle between its axis and the straight line connecting the tips, $1 / q$ is the distance between the tips. $h_{0}$ is a function of $\Omega$ and determined by the cone solution (2.1) as the maximal value of $r / \rho$, realised at the cone axis. The technical functions $\hat{w}(\varphi), B_{1}(\varphi)$ and $K(\varphi)$ are defined in eqs. (4.12), (5.12) and (5.24).

Comparing (5.27) and (4.10) we find as expected

$$
\frac{P_{2}}{q^{2}}=\frac{A_{\alpha, \Omega, q}}{2}
$$

The straightforward application of a similar conformal transformation to the hollow cones of section 3, would give us the holographic entanglement entropy of banana shaped regions with a more narrow one cutted out.

\section{Conclusions}

The main result of this paper is the explicit calculation of the regularised volume of the minimal submanifold in AdS, needed for the holographic entanglement entropy of an exemplary compact three-dimensional region $\mathcal{A}$, whose boundary has two conical singularities. As for regions with smooth boundary, the coefficient of the $1 / \epsilon^{2}$ term is equal to half of the area of the boundary $\partial \mathcal{A}$. 
The nextleading divergent term is due to the two conical singularities of our $\partial \mathcal{A}$ and as expected of squared logarithm type. Its coefficient is twice the coefficient obtained for an infinite cone with the same opening angle.

Divergences proportional to $\log \epsilon$ are present also for smooth boundaries and have conformally invariant coefficients. This invariance holds also in our case since the corresponding coefficient depends on the opening angle only.

There is however a subtlety in comparison with the infinite cone, which can be obtained as the image of our compact region $\mathcal{A}$ under an inversion on a sphere, whose center is located at one of the singular points of $\partial \mathcal{A}$. Under such exceptional conformal transformations one finds an anomaly also for smooth boundaries, as discussed in appendix B. Here we observe, that the coefficient for the banana shaped $\mathcal{A}$ is twice that for the cone plus the term $2 \pi \sin \Omega$. The factor 2 arises somehow naturally by coupling in the cone cases UV and IR regularisation by $l=1 / \epsilon$. The additive anomaly term approaches for $\Omega \rightarrow \pi / 2$ the anomaly for smooth boundaries as exemplified for spheres versus planes in appendix B.

For generic $\mathcal{A}$ in $d=3$ the finite piece of the regularised volume is not expected to be conformally invariant. Of course we observe this also in our banana case. It depends on $\Omega$ and the angle $\alpha$ between the axis of the banana and the straight line through its two conical tips. While $\Omega$ is invariant under conformal maps, $\alpha$ is not.

A second set of observations arose in connection with the following issue. Solutions of the nonlinear second order differential equation for $h(\vartheta)$, governing the minimal submanifold for the case where $\mathcal{A}$ is a cone with opening angle $2 \Omega$, have to satisfy the boundary condition $h(\Omega)=0$ and the requirement $h(0)>0$. Generation of such solutions via a Cauchy initial value problem inside $(0, \Omega)$ requires suitable fine-tuning between $h$ and $\dot{h}$. In the analogous case in $d=2$, a small perturbation of a given fine-tuned choice of Cauchy data results in a small variation of the cusp boundary conditions. In our threedimensional case perturbing the fine-tuning, results immediately in a solution satisfying $h\left(\Omega_{1}\right)=h\left(\Omega_{2}\right)=0$ for some $\Omega_{1}<\Omega_{2}$ close to $\Omega$. But these are then boundary conditions for hollow cones.

We have calculated the divergent pieces of the regularised volume relevant for the holographic entanglement entropy for regions $\mathcal{A}$ chosen as a hollow cone, parameterised by the opening angles of its inner and outer boundary. The coefficients of the $1 / \epsilon^{2}$ term and the $\log ^{2} \epsilon$ are simply equal to the sums of the corresponding terms for two full cones with angle $\Omega_{1}$ and $\Omega_{2}$, respectively. Nontrivial dependence on both angles starts with the coefficient of $\log \epsilon$.

Depending on the two angles, one has to check whether a connected submanifold, as studied in section 3 , or the disconnected one, whose two pieces each correspond to a single full cone, are favoured by its smaller volume. We have shown that to each $\Omega_{2}$ belongs a bound for $\Omega_{1}$, such that below this bound only the disconnected solution exists. Above this bound one finds two connected solutions. Among them, at least for very small $\Omega_{2}-\Omega_{1}$, the one which stays closer to the boundary of AdS is favoured. It would be interesting to fully explore the pattern of this type of geometrically induced phase transition, as well as its embedding in a setting with temperature. In connection with such an analysis also the subadditive inequality for the entanglement entropy could be of interest. ${ }^{9}$

\footnotetext{
${ }^{9}$ For an application to the cusp case in $d=2$ see $[11,12]$.
} 
Since the complement of a hollow cone consists of two regions touching each other only at the tip of the hollow cone, our observed angle dependent phase transition resembles the distance dependent transitions of the entanglement entropy for disconnected regions discussed in $[14,18,19]$.

\section{Acknowledgments}

I would like to thank Danilo Diaz for earlier discussions on entanglement entropy and George Jorjadze for numerous useful discussions over the course of this work.

\section{A Evaluation of the integrals $J_{1}$ and $J_{2}$}

Here we discuss in detail the evaluation of $J_{1}$ and $J_{2}$, defined in (5.22), for $\epsilon \rightarrow 0$. With the substitution $x=q \epsilon / h$ and

$$
x_{\epsilon}(\varphi)=\frac{1}{B_{1}(\varphi)}-q^{2} \epsilon^{2} \frac{B_{3}(\varphi)}{B_{1}^{2}(\varphi)}+\ldots
$$

we get

$$
J_{1}=\frac{2}{q^{2} \epsilon^{2}} \int_{0}^{2 \pi} d \varphi \int_{q \epsilon / h_{0}}^{x_{\epsilon}} x \log \left(1-2 x w(\theta, \varphi)+\sqrt{(1-2 x w)^{2}-4 x^{2}-4 q^{2} \epsilon^{2}}\right) d x
$$

and after expanding the integrand in $\epsilon$

$$
J_{1}=J_{11}+J_{12}+o(1)
$$

with

$$
\begin{aligned}
& J_{11}=\frac{2}{q^{2} \epsilon^{2}} \int_{0}^{2 \pi} d \varphi \int_{q \epsilon / h_{0}}^{x_{\epsilon}} x \log \left(1-2 x w(\vartheta, \varphi)+\sqrt{(1-2 x w)^{2}-4 x^{2}}\right) d x, \\
& J_{12}=-4 \int_{0}^{2 \pi} d \varphi \int_{q \epsilon / h_{0}}^{x_{\epsilon}} \frac{x d x}{\left(1-2 x w(\vartheta, \varphi)+\sqrt{(1-2 x w)^{2}-4 x^{2}}\right) \sqrt{(1-2 x w)^{2}-4 x^{2}}} .
\end{aligned}
$$

The presence of the function $\left.w(\vartheta(h), \varphi)\right|_{h=\frac{q \epsilon}{x}}$ needs some special care. We split the $x$ integration interval into

$$
I_{\text {lower }}=\left(q \epsilon / h_{0},(q \epsilon)^{\delta}\right), \quad I_{\text {upper }}=\left((q \epsilon)^{\delta}, x_{\epsilon}\right), \quad \text { with } \quad \frac{2}{3}<\delta<1 .
$$

$\left.w(\vartheta(h), \varphi)\right|_{h=\frac{q \epsilon}{x}}$ is bounded. Furthermore, in the lower interval $I_{\text {lower }}$ the variable $x$ is small and we can use

$$
\log \left(1-2 x w+\sqrt{(1-2 x w)^{2}-4 x^{2}}\right)=\log 2-2 x w+\mathcal{O}\left(x^{2}\right) .
$$

This leads to ${ }^{10}$

$$
\begin{aligned}
J_{11}= & 2 \pi \log 2\left((q \epsilon)^{2 \delta-2}-\frac{1}{h_{0}^{2}}\right)+o(1) \\
& +\frac{2}{q^{2} \epsilon^{2}} \int_{0}^{2 \pi} d \varphi \int_{(q \epsilon)^{\delta}}^{x_{\epsilon}} x \log \left(1-2 x w(\vartheta, \varphi)+\sqrt{(1-2 x w)^{2}-4 x^{2}}\right) d x .
\end{aligned}
$$

\footnotetext{
${ }^{10}$ The $\mathcal{O}\left(x^{2}\right)$ term in (A.6), together with the explicit prefactor $1 / \epsilon^{2}$, yields a contribution vanishing for $\epsilon \rightarrow 0$. This is guaranteed by the choice $\delta>2 / 3$ in (A.5).
} 
In the remaining $x$-interval $I_{\text {upper }}$ the angle $\vartheta$ is close to $\Omega$, since $h=q \epsilon / x$ is small. With (2.7), (5.4) and (4.12) we get

$$
\begin{aligned}
w(\vartheta, \varphi) & =\hat{w}(\varphi)+K(\varphi) h^{2}+\ldots \\
K(\varphi) & =\frac{1}{4}(\cos \alpha \cos \Omega+\sin \alpha \cos \Omega \cot \Omega) .
\end{aligned}
$$

Then the expansion of the logarithm in (A.7) yields

$$
\begin{aligned}
& \log \left(1-2 x w(\vartheta, \varphi)+\sqrt{(1-2 x w)^{2}-4 x^{2}}\right) \\
& \quad=\log \left(1-2 x \hat{w}(\varphi)+\sqrt{(1-2 x \hat{w})^{2}-4 x^{2}}\right)-\frac{2 x K(\varphi) h^{2}}{\sqrt{(1-2 x \hat{w})^{2}-4 x^{2}}}+\ldots .
\end{aligned}
$$

This estimate will be used for the further evaluation of (A.7), together with (A.1) and

$$
\int_{(q \epsilon)^{\delta}}^{x_{\epsilon}} d x=\left(\int_{0}^{1 / B_{1}}-\int_{0}^{(q \epsilon)^{\delta}}-\int_{x_{\epsilon}}^{1 / B_{1}}\right) d x .
$$

Using in addition the explicit integral

$$
\begin{aligned}
& \int_{0}^{\frac{1}{2(1+\hat{w})}} x \log \left(1-2 x \hat{w}+\sqrt{(1-2 x \hat{w})^{2}-4 x^{2}}\right) d x \\
& \quad=\frac{1}{8\left(1-\hat{w}^{2}\right)}-\frac{1}{16(1+\hat{w})^{2}}-\frac{\hat{w} \arccos \hat{w}}{8\left(1-\hat{w}^{2}\right)^{3 / 2}}-\frac{\log (1+\hat{w})}{8(1+\hat{w})^{2}}
\end{aligned}
$$

we get finally

$$
\begin{aligned}
J_{11}= & \frac{2}{q^{2} \epsilon^{2}} \int_{0}^{2 \pi} d \varphi\left(\frac{1}{8\left(1-\hat{w}^{2}\right)}-\frac{1}{16(1+\hat{w})^{2}}-\frac{\hat{w} \arccos \hat{w}}{8\left(1-\hat{w}^{2}\right)^{3 / 2}}-\frac{\log (1+\hat{w})}{8(1+\hat{w})^{2}}\right) \\
& +2 \int_{0}^{2 \pi} \frac{B_{3}(\varphi)}{B_{1}^{3}(\varphi)} \log (1+\hat{w}(\varphi)) d \varphi \\
& -4 \int_{0}^{2 \pi} d \varphi \int_{0}^{1 / B_{1}} \frac{K(\varphi)}{\sqrt{(1-2 x \hat{w})^{2}-4 x^{2}}} d x-\frac{2 \pi \log 2}{h_{0}^{2}}+o(1) .
\end{aligned}
$$

In the asymptotic evaluation of $J_{12}$ one performs steps analogous to the above procedure. But, since in contrast to $J_{11}$, there is no divergent $1 / \epsilon^{2}$-prefactor, the replacement of the integration interval for $x$ by $\left(0,1 / B_{1}\right)$ and of $w(\vartheta, \varphi)$ by $\hat{w}(\varphi)$ generates an $o(1)$-type error only.

Therefore, with the integral ( for $B_{1}=2(1+\hat{w})$ )

$$
\int_{0}^{1 / B_{1}} \frac{d x}{\left(1-2 x \hat{w}+\sqrt{(1-2 x \hat{w})^{2}-4 x^{2}}\right) \sqrt{(1-2 x \hat{w})^{2}-4 x^{2}}}=\frac{1}{2},
$$

we get

$$
J_{12}=-4 \pi+o(1) .
$$


Let us now turn to $J_{2}$, defined in (5.22). We again use the substitution $x=q \epsilon / h$ and expand the integrand in $\epsilon$. To handle the divergence of the integrand at $x=0$, we subtract and add $1 / x \cdot \log 2$. This means

$$
\begin{aligned}
J_{2}= & -\frac{1}{4} \int_{0}^{2 \pi} d \varphi \int_{q \epsilon / h_{0}}^{x_{\epsilon}} \frac{d x}{x} \log \left(\frac{1-2 x w(\vartheta, \varphi)+\sqrt{(1-2 x w)^{2}-4 x^{2}}}{2}\right) \\
& -\frac{1}{4} \int_{0}^{2 \pi} d \varphi \int_{q \epsilon / h_{0}}^{x_{\epsilon}} \frac{d x \log 2}{x}+o(1) .
\end{aligned}
$$

Now the first term can be handled as the integral for $J_{12}$ above. In the second term the $x$-integration is trivial, and expanding its result in $\epsilon$ we get with (A.1)

$$
\begin{aligned}
J_{2}= & -\frac{1}{4} \int_{0}^{2 \pi} d \varphi \int_{0}^{1 / B_{1}} \frac{d x}{x} \log \left(\frac{1-2 x \hat{w}(\varphi)+\sqrt{(1-2 x \hat{w})^{2}-4 x^{2}}}{2}\right) \\
& +\frac{\pi \log 2}{2} \log (q \epsilon)-\frac{\pi \log 2}{2} \log h_{0} \\
& +\frac{\log 2}{4} \int_{0}^{2 \pi} \log B_{1}(\varphi) d \varphi+o(1) .
\end{aligned}
$$

\section{B Anomaly under exceptional conformal transformations for smooth boundaries}

In ref. [16] there has been observed an anomaly under certain conformal transformations for $d=2$, see also [17]. As mentioned in the introduction, for smooth $\partial A$ in $d=2$ the finite piece of $V\left(\gamma_{A}\right)$ is conformally invariant. This invariance is broken if $\partial A$ is a compact contour passing the origin and is mapped under inversion on the unit circle to a contour extending up to infinity. The prototype is the map of circles to straight lines.

We can observe an analogous anomaly in $d=3$. There the coefficient of the logarithmic divergence is invariant under non-exceptional conformal transformations. ${ }^{11}$ Let us consider spheres $S^{2} \subset \mathbb{R}^{3}$, touching the origin, which are mapped to two-dimensional planes under inversion on the unit sphere. For the plane one gets, with an IR cut-off $l$,

$$
V_{\epsilon}^{\text {plane }}=\frac{l^{2}}{2} \frac{1}{\epsilon^{2}}
$$

On the other side, if $\partial A$ is a sphere of radius $L$, we get from [1]

$$
V_{\epsilon}^{\text {sphere }}=2 \pi L^{2} \frac{1}{\epsilon^{2}}+2 \pi \log \frac{\epsilon}{L}+\mathcal{O}(1) .
$$

Therefore we find an anomaly of $a$, the coefficient of the log-term in (1.4). It changes from zero for the planar case to $2 \pi$ in the spherical case. Remarkably, it is just the same value as for the anomaly of the finite piece in the case $d=2$.

Open Access. This article is distributed under the terms of the Creative Commons Attribution License (CC-BY 4.0), which permits any use, distribution and reproduction in any medium, provided the original author(s) and source are credited.

\footnotetext{
${ }^{11}$ We call a conformal transformation of a compact $\partial A$ exceptional if its image is noncompact.
} 


\section{References}

[1] S. Ryu and T. Takayanagi, Aspects of Holographic Entanglement Entropy, JHEP 08 (2006) 045 [hep-th/0605073] [INSPIRE].

[2] S.N. Solodukhin, Entanglement entropy of black holes, Living Rev. Rel. 14 (2011) 8 [arXiv:1104.3712] [INSPIRE].

[3] S. Ryu and T. Takayanagi, Holographic derivation of entanglement entropy from AdS/CFT, Phys. Rev. Lett. 96 (2006) 181602 [hep-th/0603001] [INSPIRE].

[4] C.R. Graham and E. Witten, Conformal anomaly of submanifold observables in AdS/CFT correspondence, Nucl. Phys. B 546 (1999) 52 [hep-th/9901021] [INSPIRE].

[5] S.N. Solodukhin, Entanglement entropy, conformal invariance and extrinsic geometry, Phys. Lett. B 665 (2008) 305 [arXiv: 0802.3117] [INSPIRE].

[6] R.C. Myers and A. Singh, Entanglement Entropy for Singular Surfaces, JHEP 09 (2012) 013 [arXiv: 1206.5225] [INSPIRE].

[7] P. Bueno and R.C. Myers, Universal entanglement for higher dimensional cones, JHEP 12 (2015) 168 [arXiv:1508.00587] [INSPIRE].

[8] S.-J. Rey and J.-T. Yee, Macroscopic strings as heavy quarks in large- $N$ gauge theory and anti-de Sitter supergravity, Eur. Phys. J. C 22 (2001) 379 [hep-th/9803001] [InSPIRE].

[9] J.M. Maldacena, Wilson loops in large-N field theories, Phys. Rev. Lett. 80 (1998) 4859 [hep-th/9803002] [INSPIRE].

[10] N. Drukker, D.J. Gross and H. Ooguri, Wilson loops and minimal surfaces, Phys. Rev. D 60 (1999) 125006 [hep-th/9904191] [INSPIRE].

[11] T. Hirata and T. Takayanagi, AdS/CFT and strong subadditivity of entanglement entropy, JHEP 02 (2007) 042 [hep-th/0608213] [INSPIRE].

[12] T. Hirata, New inequality for Wilson loops from AdS/CFT, JHEP 03 (2008) 018 [arXiv:0801.2863] [INSPIRE].

[13] I.R. Klebanov, T. Nishioka, S.S. Pufu and B.R. Safdi, On Shape Dependence and RG Flow of Entanglement Entropy, JHEP 07 (2012) 001 [arXiv:1204.4160] [INSPIRE].

[14] M.R.M. Mozaffar, A. Mollabashi and F. Omidi, Holographic Mutual Information for Singular Surfaces, JHEP 12 (2015) 082 [arXiv:1511.00244] [INSPIRE].

[15] H. Dorn, Wilson loops at strong coupling for curved contours with cusps, J. Phys. A 49 (2016) 145402 [arXiv:1509.00222] [INSPIRE].

[16] N. Drukker and D.J. Gross, An Exact prediction of $N=4$ SUSYM theory for string theory, J. Math. Phys. 42 (2001) 2896 [hep-th/0010274] [INSPIRE].

[17] H. Dorn, Exceptional conformal anomaly of null polygonal Wilson loops, JHEP 11 (2013) 063 [arXiv: 1307.6341] [INSPIRE].

[18] M. Headrick, Entanglement Renyi entropies in holographic theories, Phys. Rev. D 82 (2010) 126010 [arXiv: 1006.0047] [INSPIRE].

[19] E. Tonni, Holographic entanglement entropy: near horizon geometry and disconnected regions, JHEP 05 (2011) 004 [arXiv: 1011.0166] [INSPIRE]. 